\title{
Safety and efficacy of single-balloon enteroscopy in management of gastrointestinal bleeding in patients with a left ventricular assist device
}

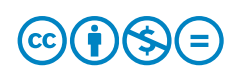

\author{
Authors \\ Abhinav Koul ${ }^{1}$, Donald M. Pham² ${ }^{2}$, Arjun Nanda ${ }^{3}$, Kevin E. Woods ${ }^{1}$, \\ Steven D. Keilin ${ }^{1}$ \\ Institutions \\ 1 Emory University School of Medicine, Department of Medicine, \\ Division of Digestive Diseases, Atlanta, Georgia, United States \\ 2 Atlanta Gastroenterology Associates, Duluth, Georgia, United \\ States \\ 3 University of Alabama at Birmingham, Department of \\ Gastroenterology and Hepatology, Birmingham, Alabama, \\ United States
}

submitted 7.1.2016

accepted after revision 5.7.2016

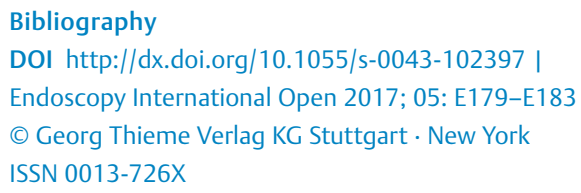

Corresponding author

Steven D. Keilin, M.D., Associate Professor of Medicine, Director, Pancreaticobiliary Service, Associate Director, Advanced Endoscopy Fellowship, Division of Gastroenterology, Emory University School of Medicine, Emory Clinic, Building B, 1365 Clifton Road NE, Atlanta, GA 30322

steven.keilin@emory.edu

\begin{abstract}
Background and study aims Left ventricular assist devices (LVADs) are currently the standard of care in treatment of patients with end-stage heart failure waiting for heart transplant as well as destination therapy for non-transplant candidates. However, patients with LVADs are at increased risk of gastrointestinal bleeding due to the device's unique effects on hemodynamics. A major source of gastrointestinal bleeding in these patients are gastrointestinal angioectasias located within the small bowel that can only be reached with deep enteroscopy. The goal of our study was to determine the safety and efficacy of single-balloon enteroscopy (SBE) in treating gastrointestinal bleeding in patients with LVADs.

Patients and methods We present a retrospective case series performed on patients with LVADs who underwent SBE to treat episodes of gastrointestinal bleeding. All procedures were performed at Emory University Hospital by a single endoscopist. Patient demographics, diagnosis and treatment of gastrointestinal bleeding, episodes of re-bleeding, and procedure-related complications were examined.

Results A total of 27 SBE procedures performed in 14 patients were reviewed. SBE was performed in an antegrade approach in $89 \%(24 / 27)$ of cases. Deep intubation was achieved in all antegrade procedures, with the distal jejunum reached in $79 \%$ (19/24) of cases. The diagnostic yield was $78 \%$. There were no reported complications associated with the procedures.

Conclusions SBE is a safe and effective modality to manage gastrointestinal bleeding in patients with LVADs.
\end{abstract}

\section{Introduction}

Left ventricular assist devices (LVADs) are currently considered the standard of care in patients with chronic, end-stage heart failure who have failed medical therapy [1]. The HeartMate II LVAD (HMII) and the Jarvik 2000 LVAD are the 2 most commonly used continuous flow pumps in the United States [2]. Because LVADs can prolong survival in patients with end stage heart failure, they are being increasingly used as destination therapy in patients who are not transplant candidates. However, LVAD therapy is associated with many adverse events (AEs) including infection, arrhythmia, thrombosis, and gastrointestinal bleeding. Of these, gastrointestinal bleeding in patients with an LVAD is particularly difficult to manage and can be associated with significant cost. The incidence of gastrointestinal bleeding has been studied in patients with the HMII and Jarvik 2000 LVAD, and was reported to be approximately 20 to $40 \%$ [1,3-7].

Arteriovenous malformations (AVMs) and gastrointestinal angiodysplasias have been identified as the source of gastrointestinal bleeding in many cases involving patients with an LVAD $[3,4]$. The etiology of gastrointestinal bleeding in these patients is multifactorial, with several proposed mechanisms contributing to this phenomenon [5]. Among LVAD patients, up to $30 \%$ of angiodysplasias and AVMs occur in the small intestine and can be difficult to detect with conventional endoscopy [3, 6]. While modalities such as capsule endoscopy can be useful in identifying bleeding in the small bowel, interventions such as balloon enteroscopy have the dual benefit of diagnosis and potential therapeutic intervention. Performing deep enteroscopy 
- Table 1 Characteristics of patients with left ventricular assist devices undergoing single-balloon enteroscopy (SBE) for gastrointestinal bleeding.

\begin{tabular}{|l|l|}
\hline Total patients & 14 \\
\hline Male & $93 \%$ \\
\hline Average Age & $64.5(39-78)$ \\
\hline ASA class 4 & $86 \%$ \\
\hline LVAD types & \\
\hline - HeartMate II & $93 \%$ \\
\hline - Terumo Duraheart & $7 \%$ \\
\hline Average hematocrit prior to SBE & $24.7(18-31.5)$ \\
\hline LVAD, left ventricular assist device & \\
\hline
\end{tabular}

in patients with LVADs can be challenging, as this particular group of patients is at high risk for peri-procedural AEs due to sedation difficulties and hemodynamic instability. Individual case reports have described double balloon enteroscopy (DBE) being used successfully to treat gastrointestinal bleeding in patients with LVADs $[8,9]$. A more recent case series reviewed 10 patients with LVADs who underwent DBE for treatment of gastrointestinal bleeding with a diagnostic yield of $69 \%$ and a therapeutic yield of $89 \%$ [10]. None of these reports described any procedure-related AEs.

Single balloon enteroscopy (SBE) is an endoscopic modality typically used to diagnose and treat small intestinal diseases that cannot be reached with standard endoscopy, colonoscopy or push enteroscopy. SBE remains an attractive option compared to other endoscopic modalities such as DBE in that it can achieve a similar depth of small bowel insertion in less time and only requires a single operator. Our study aimed to determine the safety and efficacy of SBE in treating gastrointestinal bleeding in patients with an LVAD. This is the largest study to date detailing the safety and efficacy of SBE in LVAD patients.

\section{Patients and methods}

This study was approved by the Institutional Review Board at the Emory University School of Medicine. We conducted a retrospective case series of all adult patients with LVADs who underwent SBE between February 2009 and May 2014. The indications for SBE included any clinical evidence of gastrointestinal bleeding such as iron deficiency anemia, symptomatic anemia, hematemesis, melena, hematochezia and/or a positive fecal occult blood test. Exclusion criteria included patients who had a history of gastrointestinal bleeding prior to LVAD placement. Patient demographics, diagnosis and treatment of gastrointestinal bleeding, episodes of re-bleeding, and procedure related AEs were recorded.

All SBE procedures were performed at Emory University Hospital (EUH) during the study period by one endoscopist (S. K.) experienced with the procedure. All patients had either general anesthesia or monitored anesthesia care (MAC) during the procedure. The anesthetic agent used for MAC cases varied and

- Table 2 Video capsule endoscopy findings from patient group ${ }^{1}$.

Positive capsule endoscopy

- Bleeding

- AVM

- Polyp

Negative capsule endoscopy

No capsule endoscopy performed

AVM, arteriovenous malformation

${ }^{1}$ All findings were in the small bowel.

was administered at the discretion of the anesthesiologist involved in the case. In addition, specialized LVADtechnicians were present during all procedures and blood pressure was monitored with a Terumo cuff. Patients with Automatic Implantable Cardioverter Defibrillators (AICDs) had their devices interrogated before and after SBE by Cardiology. Those patients also had a magnet placed on the AICD when electrocautery was used during SBE. Single-balloon enteroscopy was performed using the small intestine enteroscope (Olympus SIF type Q180) and splinting tube (Olympus ST-SB1) attached to a balIoon control unit. Carbon dioxide insufflation was used in all cases. The depth of insertion was determined by the endoscopist, though this was subjective and not standardized. No fluoroscopy was used during the procedures. The diagnosis of small bowel pathology and treatment of lesions were done at the discretion of the endoscopist.

Because a majority of the patients were outside referrals from other advanced heart failure and heart transplant centers, follow-up data were obtained by directly contacting each referring physician and hospital to obtain complete medical records including discharge summaries as well as endoscopic and laboratory data. Based on these results, the number of re-bleeding episodes and repeat endoscopies after the index SBE were determined. All patients included in the study had a recent negative esophagogastroduodenoscopy (EGD) and colonoscopy prior to undergoing SBE. In addition, 12 of the 14 patients included in the study underwent capsule endoscopy prior to SBE. Those individuals who had capsule endoscopy done at an outside institution had their images reviewed at EUH.

\section{Results}

During the study period, there were a total of 27 SBE procedures performed on 14 patients with LVADs who had clinical evidence of gastrointestinal bleeding. The majority of patients $(12 / 14)$ had LVADs as destination therapy for their heart failure. Thirteen patients (93\%) were male, with a mean age of 64.5 years (range $39-78$ ), and $86 \%$ of the patients were ASA class 4 ( $\downarrow$ Table 1). All procedures were done at the endoscopy unit in the hospital. A majority of patients (72\%) had an abnormal video capsule endoscopy (VCE) prior to the SBE. Two patients had a negative VCE and 2 did not have a prior VCE ( $>$ Table 2 ). The 2 patients in whom VCE was deferred presented with significant anemia and melena, therefore SBE was performed directly 
- Table 3 Indications for performing single-balloon enteroscopy in patient group.

\begin{tabular}{|l|l|}
\hline Anemia only & $4 / 14(29 \%)$ \\
\hline Anemia + melena & $9 / 14(64 \%)$ \\
\hline Anemia + melena + hematochezia & $1 / 14(7 \%)$ \\
\hline
\end{tabular}

after a negative EGD and colonoscopy due to their clinical presentation with considerable overt bleeding. The average hematocrit prior to endoscopy was 24.7 units (range 18-31.5). SBE was performed in the setting of overt bleeding in $71 \%(10 / 14)$ of patients ( $\triangleright$ Table 3 ).

The majority of the procedures were done in the hospital endoscopy unit at Emory University Hospital (25/27), with monitored anesthesia care in $70 \%$ (19/27) of cases. The antegrade approach was performed in $89 \%$ (24/27) of cases, and there were 2 patients who had both an antegrade and retrograde SBE during the same session. The average endoscopic procedural time was 37 minutes (range 24-69 min). Intubation of the distal jejunum was achieved in $79 \%$ (19/24) of antegrade cases ( $\vee$ Table 4$)$. The terminal ileum was reached in $66 \%(2 / 3)$ of retrograde procedures, with one procedure aborted due to a surgical anastomotic stricture in the colon that prevented passage of the endoscope.

Of the 27 SBEs performed, 21 procedures revealed lesions that accounted for gastrointestinal bleeding, for a diagnostic yield of $78 \%$. The majority of cases (67\%) showed both bleeding and non-bleeding angioectasias ( $>$ Fig. 1). However other findings included ulcers in two cases, as well as a polyp that demonstrated adenocarcinoma on pathology ( $>$ Table 5 ). The majority of lesions were successfully treated with argon plasma coagulation (APC) in 17/21 (80\%) of the procedures. However other interventions were utilized alone or in combination with APC in 4 other cases ( $>$ Table 6 ). There were no reported AEs associated with the procedures.

The average follow-up period in these patients was 79 days (range 3-350). Subsequent to the index SBE, patients had on average one episode of re-bleeding (range $0-5$ episodes), with 5 patients having 2 or more episodes. Additionally, patients also had, on average, 2 endoscopic procedures following the index SBE (range 0-7 additional procedures), with 5 patients having 3 or more procedures. One patient required an additional 3 SBEs and another patient required 5 additional SBEs for bleeding. In patients who underwent more than one SBE, the average time between procedures was about 117 days (range 14-350 days). One patient died during the followup period from AEs related to his heart disease.

\section{Discussion}

As the use of LVADs becomes more common among those with end-stage heart failure, gastrointestinal bleeding will also increase and these patients will undoubtedly require endoscopy to isolate and treat the bleeding source. Endoscopic interventions in LVAD patients can be challenging due to their unique hemodynamics, overall comorbidities, and need for anticoagu-

- Table4 General characteristics of single-balloon enteroscopy procedures.

Total number of procedures

27

- Antegrade $89 \%$

\begin{tabular}{l|l} 
- Distal jejunum intubated & $79 \%$ \\
\hline
\end{tabular}

- Retrograde

$11 \%$

Locations of procedures

- Inpatient gastrointestinal endoscopy unit

$93 \%$

- Operating room

$7 \%$

Type of anesthesia

- Monitored anesthesia care $70 \%$

- General anesthesia $30 \%$

Average time of procedure (min)

Adverse events

$37(24-69)$

None

lation. In addition many patients bleed from angioectasias in the small bowel that cannot be reached by a standard endoscope [11]. While there is evidence that conventional endoscopy and capsule endoscopy can be safe in LVAD patients [12], there are limited data on safety and efficacy of deep enteroscopy in this patient population.

This is the largest study to date on use of SBE to treat gastrointestinal bleeding in LVADpatients. We demonstrated that deep enteroscopy with SBE is safe in a group of patients that is predominantly ASA class 4 . We did not record any major hemodynamic changes in patients during any of the procedures, and there were no documented complications. There was no interaction between electric cautery and the LVAD during our cases. The majority of cases were completed in the endoscopy unit at the hospital under MAC, which is less costly than general anesthesia. We found SBE to be effective in achieving deep small bowel intubation in all patients. Depth of insertion, procedure time, and amount of anesthesia used during our procedures were not significantly different than in standard SBE cases in patients without LVADs, although no formal statistical analysis was performed to corroborate these findings. SBE also had a high diagnostic yield and allowed therapies to be performed on small bowel lesions beyond the reach of conventional endoscopy.

Our study does have important limitations as the true number of re-bleeding episodes and additional endoscopic procedures might be under reported since we relied on outside follow-up data for this information. In addition, the length of follow-up was not consistent among all patients because the majority of our patients were referrals from other institutions and it was challenging to obtain this information retrospectively. In patients who are not transplant candidates and use LVADs as destination therapy, as was the case in the majority of our patients, there will always be a potential for additional episodes of gastrointestinal bleeding as a direct result of the LVAD itself. These patients will also likely remain anemic due to hemolysis caused by the LVAD as well as from anemia of chronic disease. 

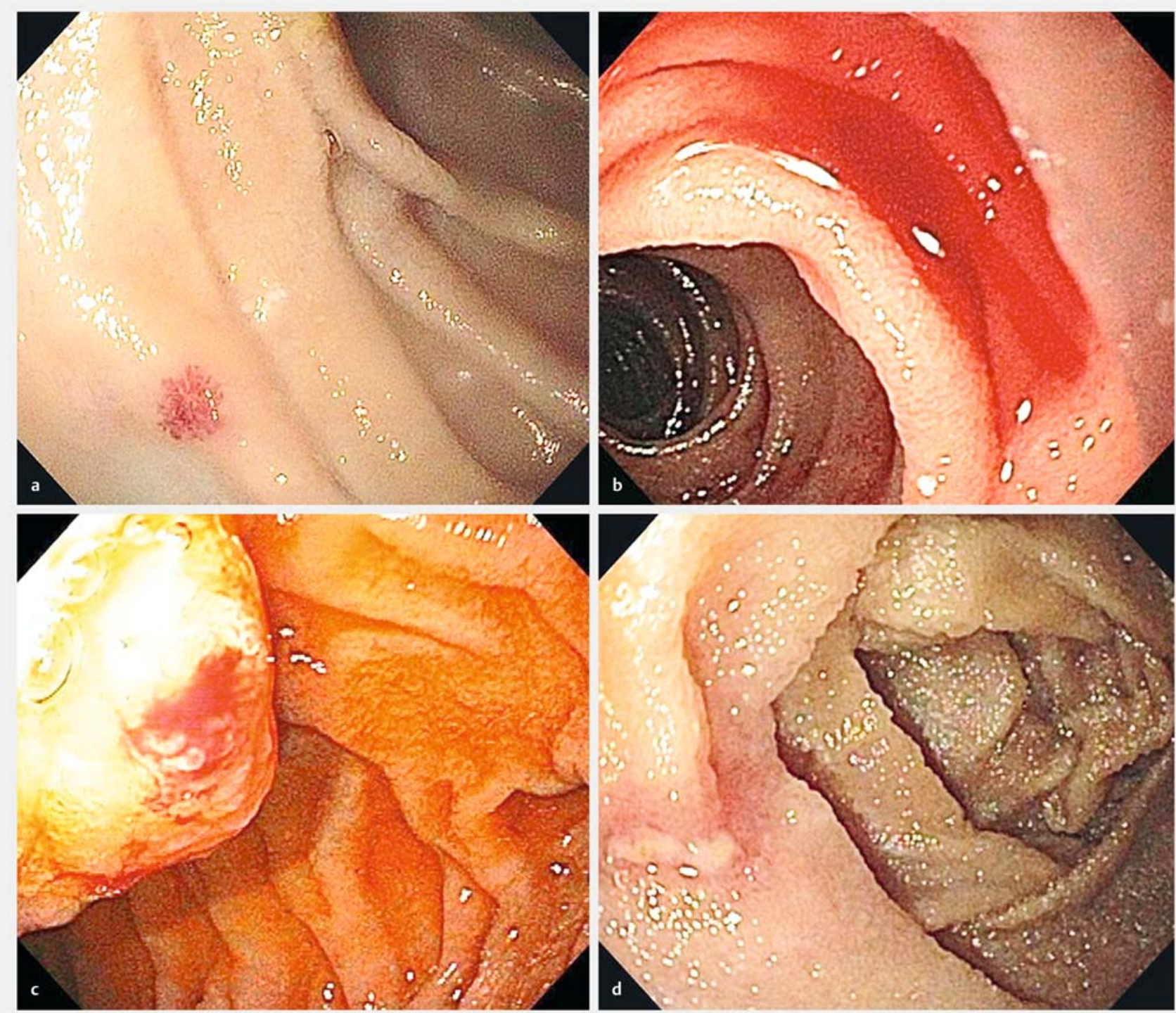

Fig. 1 Pictures of lesions seen in the small bowel during SBE cases. Clockwise from top left corner: a An AVM. b Active bleeding in the small bowel. c An ulcer. d A polyp, later discovered to be adenocarcinoma.

- Table 5 Findings from single-balloon enteroscopy (SBE) procedures.

\begin{tabular}{|l|l|}
\hline Non-bleeding AVM only & $12 / 27(44 \%)$ \\
\hline Bleeding AVM only & $2 / 27(7 \%)$ \\
\hline Non-bleeding + bleeding AVMs & $4 / 27(15 \%)$ \\
\hline Ulcer only & $1 / 27(4 \%)$ \\
\hline Ulcer + non-bleeding AVM & $1 / 27(4 \%)$ \\
\hline Polyp & $1 / 27(4 \%)$ \\
\hline Normal SBE & $6 / 27(22 \%)$ \\
\hline
\end{tabular}

AVM, arteriovenous malformation; SBE, single-balloon enteroscopy
- Table 6 Interventions performed during single-balloon enteroscopy procedures.

\begin{tabular}{|l|c|}
\hline APC only & $17 / 21(80 \%)$ \\
\hline Epinephrine only & $1 / 21(5 \%)$ \\
\hline APC + epinephrine & $1 / 21(5 \%)$ \\
\hline APC + hemoclip & $1 / 21(5 \%)$ \\
\hline Snare polypectomy & $1 / 21(5 \%)$ \\
\hline APC, argon plasma coagulation & \\
\hline
\end{tabular}


Therefore we can assume that multiple factors contribute to anemia in patients with LVADs and they are also at high risk of re-bleeding that will require additional endoscopic procedures after SBE. Going forward, it would be advantageous to have more structured guidelines to follow these patients prospectively in order to better evaluate the long-term clinical efficacy of SBE in this subset of patients. We are currently using the data gained from this study to develop such an algorithm at our institution and identify the optimal role for SBE in treating gastrointestinal bleeding in patients with LVADs. Other studies comparing SBE and DBE in LVAD patients as well as the efficacy of SBE versus standard endoscopy and/or medical management in patients with LVADs are possible areas of future research.

\section{Conclusions}

In summary, SBE is a safe and effective modality to control gastrointestinal bleeding in patients with an LVAD. It can be performed in a sick patient population under MAC in a general gastrointestinal unit without requiring general anesthesia or an operating room. Additional research is needed to further validate the use of SBE within the algorithm to treat gastrointestinal bleeding in LVAD patients. Our study shows that at this time, SBE is a useful tool for the gastroenterologists in this extremely challenging patient population.

\section{Competing interests}

None

\section{References}

[1] Morgan JA, Paone G, Nemeh HW et al. Gastrointestinal bleeding with the HeartMate II left ventricular assist device. J Heart Lung Transplant 2012; 31: 715-718

[2] Suarez J, Patel CB, Felker GM et al. Mechanisms of bleeding and approach to patients with axial-flow left ventricular assist devices. Circ Heart Fail 2011; 4: 779-784

[3] Demirozu ZT, Radovancevic R, Hochman LF et al. Arteriovenous malformation and gastrointestinal bleeding in patients with the HeartMate II left ventricular assist device. J Heart Lung Transplant 2011; 30 : $849-853$

[4] Aggarwal A, Pant R, Kumar S et al. Incidence and management of gastrointestinal bleeding with continuous flow assist devices. Ann Thorac Surg 2012; 93: 1534 - 1540

[5] Harvey L, Holley CT et al. Gastrointestinal bleed after left ventricular assist device implantation: incidence, management, and prevention. Ann Cardiothorac Surg 2014; 3: 475-479

[6] Stern DR, Kazam J, Edwards P et al. Increased incidence of gastrointestinal bleeding following implantation of the HeartMate II LVAD. J Card Surg 2010; 25: 352-356

[7] Letsou GV, Shah N, Gregoric ID et al. Gastrointestinal bleeding from arteriovenous malformations in patients supported by the Jarvik 2000 axial-flow left ventricular assist device. J Heart Lung Transplant 2005; 24: $105-109$

[8] Decker GA, Miller ED, Pasha SF et al. Deep enteroscopy in patients with left ventricular assist devices: practical and technical considerations. Endoscopy 2010; 42: (Suppl. 02): E194

[9] Tarzia V, Dal Lin C, Bottio T et al. Occult gastrointestinal bleeding in patients with a left ventricular assist device axial flow pump: diagnostic tools and therapeutic algorithm. J Thorac Cardiovasc Surg 2012; 143: e28-31

[10] Edwards AL, Monkemuller K, Pamboukian SL et al. Utility of doubleballoon enteroscopy in patients with left ventricular assist devices and obscure overt gastrointestinal bleeding. Endoscopy 2014; 46: $986-988$

[11] Sarosiek K, Bogar L et al. An old problem with a new therapy: gastrointestinal bleeding in ventricular assist device patients and deep overtube-assisted enteroscopy. ASAIO J 2013; 59: 384-389

[12] Kushir VM, Sharma S, Ewald GA et al. Evaluation of gastrointestinal bleeding after implantation of left ventricular assist device. Gastrointest Endosc 2012; 75: $973-979$ 